Proceedings of the 2009 Winter Simulation Conference

M. D. Rossetti, R. R. Hill, B. Johansson, A. Dunkin, and R. G. Ingalls, eds.

\title{
SENSITIVITY ANALYSIS FOR BARRIER OPTIONS
}

\author{
Yongqiang Wang \\ Department of Electrical and Computer Engineering \\ \& Institute for Systems Research \\ University of Maryland \\ College Park, MD, 20742, U.S.A
}

\author{
Michael C. Fu \\ The Robert H. Smith School of Business \\ \& Institute for Systems Research \\ University of Maryland \\ College Park,MD, 20742, U.S.A
}

Steven I. Marcus

Department of Electrical and Computer Engineering

\& Institute for Systems Research

University of Maryland

College Park, MD, 20742, U.S.A

\begin{abstract}
Barrier options are popular derivative securities with payoffs dependent on whether or not an underlying asset crosses a barrier. This paper presents a Monte Carlo simulation-based method of sensitivity analysis for barrier options based on smoothed perturbation analysis (SPA) for a general form of discontinuous sample function payoffs. The connection between the resulting SPA estimator and the probability formula derived in Hong (2008) and its generalization in Liu and Hong (2009) is explored. Using a Brownian bridge result, the estimator is applied to continuously-monitored barrier options with rebates. Illustrative simulation examples are provided.
\end{abstract}

\section{INTRODUCTION}

Since its introduction to finance by Boyle (1977), Monte Carlo simulation has become one of the standard methods for option pricing due to the increasing complexity of derivative securities. Gradient estimation plays a central role in the hedging and pricing of various security derivatives. For example, Fu and $\mathrm{Hu}$ (1995) price American call options with discrete dividends by parameterizing the exercise boundary and using an SPA gradient estimator in a stochastic approximation algorithm to maximize the expected payoff with respect to the parameters. Perhaps even more importantly than for option pricing, gradients are central for hedging risk. For example, the first-order derivatives of the stock price with respect to the price and volatility, called the "delta" and "vega," respectively, are used to hedge against price and volatility swings. This paper considers sensitivity analysis for continuously-monitored barrier options, which are a special type of options whose payoff depends on if the underlying security price crosses a specific barrier during the life of the option. There are two main types of barrier options: knock-out options, which pay zero if a barrier crossing occurs, and knock-in options, which have zero payoff unless there is a barrier crossing. For more information on barrier options pricing, see Glasserman and Staum (2001), Broadie, Glasserman, and Kou (1997).

In most cases, there are no analytical solutions for the gradient, and Monte Carlo simulation is frequently adopted to estimate the gradient (Broadie and Glasserman 1996, Fu and $\mathrm{Hu}$ 1995). In the simulation literature, gradient estimation has been studied extensively (Fu 2006). Techniques include infinitesimal perturbation analysis (IPA) (Ho and Cao 1991, Glasserman 1991), the likelihood ratio method (Reiman and Weiss 1989), finite differences, and Malliavin calculus (Fournié et al. 2001, Chen and Glasserman 2007).

For a general introduction to IPA, refer to Ho and Cao (1991), Glasserman (1991). The IPA method explores properties of sample paths and usually gives estimators with smaller variance compared with other estimators. The key problem of 
Wang, Fu and Marcus

IPA is the interchangeability of differentiation and integration, which is not applicable if the sample performance function is discontinuous. By using conditional expectation, smoothed perturbation analysis (SPA) (Gong and Ho 1987, Fu and Hu 1997) can overcome this difficulty. To cope with the discontinuities in payoff functions, it may be difficult to choose on what to condition, which is viewed as a drawback of SPA, but we will illustrate with examples how to do this for a certain class of discontinuous sample performance functions. The likelihood ratio (LR) method, also called the score function (SF) method (Rubinstein and Melamed ), does not require the sample performance function to be continuous, and the interchangeability of differentiation and integration is often not a problem, although the variance can be high.

Recently, for the purpose of estimating quantile sensitivities, Hong (2008) derived a probability formula that is generalized in Liu and Hong (2009) to derive gradient estimators for performance functions containing indicator functions. In this paper, we explore the connection between the SPA approach and the method in Hong (2008). Specifically, we consider performance functions with an indicator function and use the SPA approach to derive gradient estimators that match those in Liu and Hong (2009), but are valid under different conditions. More generally, SPA can also be used to deal with the sensitivity analysis for performance functions with more complicated forms. We give a pricing algorithm for barrier options with underlying securities following general diffusion processes. The payoff function in our pricing algorithm involves multiple indicator functions and has a complicated form, and SPA is used to obtain gradient estimators for the barrier option. We also extend our result to barrier options with rebates. Finally numerical examples are given to demonstrate the effectiveness of our algorithm.

\section{PROBLEM SETTING}

We consider the sensitivity analysis problem for a knock-out option with the underlying financial security following a stochastic process $\left\{S_{t}, t \in[0, T]\right\}$. We only deal with European-style options, which can only be exercised at maturity. Continuously-monitored barrier options will be considered. The state vector of the underlying security at the simulated discretized points $\left(t_{1}, t_{2}, \cdots, t_{m}=T\right)$, is denoted as $\left(S_{1}, S_{2}, \cdots, S_{m}\right)$ (abbreviating the more explicit $\left(S_{t_{1}}, S_{t_{2}}, \cdots, S_{t_{m}}\right)$ ). For simplicity, we also assume that the length of the time between two consecutive sampled dates $t_{i+1}$ and $t_{i}$ is a constant $\Delta t$.

\subsection{Examples}

\subsubsection{Geometric Brownian Motion}

In this example, the stock price $S_{t}$ is governed by the dynamics

$$
d S_{t}=r S_{t} d t+\sigma S_{t} d B_{t}
$$

where $\left\{B_{t}\right\}$ is the standard Brownian motion process, $r$ is the riskless interest rate, and $\sigma$ is the volatility. This model has an exact discretization solution

$$
S_{i+1}=S_{i} \exp \left(\left(\mu-\frac{1}{2} \sigma^{2}\right) \Delta t+\sigma \sqrt{\Delta t} Z_{i}\right),
$$

where $Z_{1}, Z_{2}, \cdots, Z_{m-1}$ are i.i.d standard normal random variables. For a down-and-out barrier call option, the barrier is a price level $H<S_{0}$ and the barrier option has a discounted payoff

$$
V=\exp (-r T)\left(S_{m}-K\right)^{+} 1_{\left\{\inf _{t \leq T} S_{t}>H\right\}}
$$

where $K$ is the strike price, and $t_{m}=T$ the maturity date. For this simple model, there exist closed-form solutions for the pricing problem. We are interested in the sensitivity analysis of the expected payoff function $E[V]$ with respect to parameter $\theta$, which could be $\mu, \sigma, K, S_{0}, H$. The sensitivity of $E[V]$ with respect to $H$ is defined as

$$
\frac{\partial E[V]}{\partial H}=\lim _{\varepsilon \rightarrow 0} \frac{E\left[\exp (-r T)\left(S_{m}-K\right)^{+} 1_{\left\{\inf _{t \leq T} S_{t}>H+\varepsilon\right\}}\right]-E\left[\exp (-r T)\left(S_{m}-K\right)^{+} 1_{\left\{\inf _{t \leq T} S_{t}>H-\varepsilon\right\}}\right]}{2 \varepsilon} .
$$


Wang, Fu and Marcus

\subsubsection{General Diffusion Processes}

Here we consider general diffusion processes, for example, the mean reverting Ornstein-Uhlenbeck process

$$
d S_{t}=\theta\left(\mu-S_{t}\right) d t+\sigma d B_{t}
$$

where $\theta, \mu, \sigma$ are parameters. More generally, we consider the following diffusion process

$$
d S_{t}=\mu\left(S_{t}, t\right) d t+\sigma\left(S_{t}, t\right) d B_{t}
$$

where $\mu\left(S_{t}, t\right)$ and $\sigma\left(S_{t}, t\right)$ are two functions depending on $S_{t}$ and $t$. The Ornstein-Uhlenbeck process (1) is a special case of (2). We can easily simulate the above process by Euler scheme as follows:

$$
S_{i+1}=S_{i}+\mu\left(S_{i}, t_{i}\right) \Delta t+\sigma\left(S_{i}, t_{i}\right) \sqrt{\Delta t} Z_{i+1}
$$

Since there is no analytical solution for the pricing of barrier options with underlying securities of the above form, we can use Monte Carlo simulation to estimate the option price. In this paper we are interested in the sensitivity analysis of barrier options following the above model.

\subsection{Pathwise Gradient Estimator By SPA}

Note that the payoff function of barrier options can be written in a more general form

$$
p(\theta)=E\left[\psi(\theta) 1_{\{v(\theta) \geq 0\}}\right]
$$

for random variables $\psi$ and $v$ depending on parameter $\theta$. Since there is an indicator function in the payoff function of barrier options, the classical pathwise gradient estimation technique of infinitesimal perturbation analysis (IPA) is not applicable (Glasserman 2004). Smoothed Perturbation Analysis (SPA) is a gradient estimation technique that can be used to smooth out the discontinuity in the payoff function (Gong and Ho 1987, Fu and $\mathrm{Hu}$ 1997). Recently, Hong (2008) proposed an approach to circumvent this difficulty when differentiating the expectation of an indicator function. Liu and Hong (2009) extends the method in Hong (2008) to more general cases, which is applied to obtain Greeks for financial options. Actually there is a connection between SPA and the method proposed by Hong (2008). Based on the SPA method, we give a new derivation of the gradient estimator provided in Liu and Hong (2009).

We use the following assumptions to establish the unbiasedness of pathwise estimators for functions of the form (4), where we assume $v(\theta)$ is a random variable that is expressible as a function of another random variable, in which we show explicit dependence by writing $v(\theta, \omega)$.

Assumption 1. For $\omega$ a continuous-valued random variable, $v(\theta, \omega)$ is a random variable that is differentiable w.r.t. $\theta$ almost everywhere, i.e.

$$
v^{\prime}(\theta, \omega)=\lim _{\Delta \theta \rightarrow 0} \frac{v(\theta+\Delta \theta, \omega)-v(\theta, \omega)}{\Delta \theta} \text { w.p.1, }
$$

and $v(\theta)$ has probability density function (p.d.f.) $f_{\theta}$.

Assumption 2. $\psi(\theta)$ is differentiable w.p.1, and there exists a random variable $\lambda_{\psi(\theta)}$ with $E\left[\lambda_{\psi(\theta)}\right]<\infty$ such that $|\psi(\theta+\Delta \theta)-\psi(\theta)| \leq \lambda_{\psi(\theta)}|\Delta \theta|$ for sufficiently small $\Delta \theta$.

Theorem 1. If $v(\cdot)$ and $\psi(\cdot)$ satisfy Assumptions 1 and 2 (respectively), then

$$
\frac{\partial}{\partial \theta} E\left[\psi(\theta) 1_{\{v(\theta) \geq 0\}}\right]=E\left[\psi^{\prime}(\theta) 1_{\{v(\theta)\} \geq 0}\right]+f_{v(\theta)}(0) E\left[\psi(\theta) v^{\prime}(\theta) \mid v(\theta)=0\right] .
$$

Proof. Given a specific $\theta$, assume there exists an $\omega^{\star}$ in the sample space $\Omega$ such that $v\left(\theta, \omega^{\star}\right)=0$; otherwise the IPA gradient estimator would be unbiased, i.e., the second term in (6) would be 0 . We consider the perturbation of $\theta$ to $\theta+\Delta \theta$, for small $\Delta \theta$. 


\section{Wang, Fu and Marcus}

First, if $v\left(\theta+\Delta \theta, \omega^{\star}\right)$ and $v\left(\theta-\Delta \theta, \omega^{\star}\right)$ always have the same sign, we will have

$$
\frac{\partial}{\partial \theta} E\left[\psi(\theta) 1_{\{v(\theta) \geq 0\}}\right]=\lim _{\Delta \theta \rightarrow 0} \frac{E\left[\psi(\theta+\Delta \theta) 1_{\{v(\theta+\Delta \theta) \geq 0\}}-\psi(\theta-\Delta \theta) 1_{\{v(\theta-\Delta \theta) \geq 0\}}\right]}{2 \Delta \theta}=E\left[\psi^{\prime}(\theta) 1_{\{v(\theta)\} \geq 0}\right]
$$

by the dominated convergence theorem. Note that we also have

$$
\frac{\partial v\left(\theta, \omega^{\star}\right)}{\partial \theta}=0
$$

Hence the theorem is proved for this case.

Now assume that $v\left(\theta+\Delta \theta, \omega^{\star}\right)$ and $v\left(\theta-\Delta \theta, \omega^{\star}\right)$ have different signs. Without loss of generality, we assume that $v\left(\theta+\Delta \theta, \omega^{\star}\right) \geq 0$ and $v\left(\theta-\Delta \theta, \omega^{\star}\right) \leq 0$. Define

$$
\mathscr{B}\left(\omega^{\star}, \theta, \Delta \theta\right)=\left\{\omega: 0 \leq v(\theta+\Delta \theta, \omega) \leq v\left(\theta+\Delta \theta, \omega^{\star}\right) ; 0 \geq v(\theta-\Delta \theta, \omega) \geq v\left(\theta-\Delta \theta, \omega^{\star}\right)\right\} .
$$

Let $\mathscr{B}^{c}\left(\omega^{\star}, \theta, \Delta \theta\right)$ be the complement set of $\mathscr{B}\left(\omega^{\star}, \theta, \Delta \theta\right)$. Then we have

$$
\begin{aligned}
\frac{\partial}{\partial \theta} E\left[\psi(\theta) 1_{\{v(\theta) \geq 0\}}\right] & =\lim _{\Delta \theta \rightarrow 0} \frac{E\left[\psi(\theta+\Delta \theta) 1_{\{v(\theta+\Delta \theta) \geq 0\}}-\psi(\theta-\Delta \theta) 1_{\{v(\theta-\Delta \theta) \geq 0\}}\right]}{2 \Delta \theta} \\
& =\Pi_{1}+\Pi_{2},
\end{aligned}
$$

where

$$
\begin{aligned}
& \Pi_{1}=\lim _{\Delta \theta \rightarrow 0} \frac{E\left[\psi(\theta+\Delta \theta) 1_{\{v(\theta+\Delta \theta) \geq 0\}}-\psi(\theta-\Delta \theta) 1_{\{v(\theta-\Delta \theta) \geq 0\}} \mid \mathscr{B}^{c}\left(\omega^{\star}, \theta, \Delta \theta\right)\right] P\left(\mathscr{B}^{c}\left(\omega^{\star}, \theta, \Delta \theta\right)\right)}{2 \Delta \theta}, \\
& \Pi_{2}=\lim _{\Delta \theta \rightarrow 0} \frac{E\left[\psi(\theta+\Delta \theta) 1_{\{v(\theta+\Delta \theta) \geq 0\}}-\psi(\theta-\Delta \theta) 1_{\{v(\theta-\Delta \theta) \geq 0\}} \mid \mathscr{B}\left(\omega^{\star}, \theta, \Delta \theta\right)\right] P\left(\mathscr{B}\left(\omega^{\star}, \theta, \Delta \theta\right)\right)}{2 \Delta \theta} .
\end{aligned}
$$

Note that

$$
\begin{aligned}
\Pi_{2} & =\lim _{\Delta \theta \rightarrow 0} \frac{E\left[\psi(\theta+\Delta \theta) 1_{\{v(\theta+\Delta \theta) \geq 0\}}-\psi(\theta-\Delta \theta) 1_{\{v(\theta-\Delta \theta) \geq 0\}} \mid \mathscr{B}\left(\omega^{\star}, \theta, \Delta \theta\right)\right] P\left(\mathscr{B}\left(\omega^{\star}, \theta, \Delta \theta\right)\right)}{2 \Delta \theta} \\
& =\lim _{\Delta \theta \rightarrow 0} E\left[\psi(\theta+\Delta \theta) 1_{\{v(\theta+\Delta \theta) \geq 0\}} \mid \mathscr{B}\left(\omega^{\star}, \theta, \Delta \theta\right)\right] \lim _{\Delta \theta \rightarrow 0} \frac{P\left(\mathscr{B}\left(\omega^{\star}, \theta, \Delta \theta\right)\right)}{2 \Delta \theta} \\
& =E\left[\psi(\theta) 1_{\{v(\theta) \geq 0\}} \mid \omega^{\star}\right] \lim _{\Delta \theta \rightarrow 0} \frac{\int_{0}^{v\left(\theta+\Delta \theta, \omega^{\star}\right)} f_{\theta+\Delta \theta}(t) d t+\int_{v\left(\theta-\Delta \theta, \omega^{\star}\right)}^{0} f_{\theta-\Delta \theta}(t) d t}{2 \Delta \theta}
\end{aligned}
$$

and that

$$
\begin{aligned}
\int_{v\left(\theta-\Delta \theta, \omega^{\star}\right)}^{v\left(\theta+\Delta \theta, \omega^{\star}\right)} \inf _{\hat{\theta} \in(\theta-\Delta \theta, \theta+\Delta \theta)} f_{\hat{\theta}}(t) d t & \leq \int_{0}^{v\left(\theta+\Delta \theta, \omega^{\star}\right)} f_{\theta+\Delta \theta}(t) d t+\int_{v\left(\theta-\Delta \theta, \omega^{\star}\right)}^{0} f_{\theta-\Delta \theta}(t) d t \\
& \leq \int_{v\left(\theta-\Delta \theta, \omega^{\star}\right)}^{v\left(\theta+\Delta \theta, \omega^{\star}\right)} \sup _{\hat{\theta} \in(\theta-\Delta \theta, \theta+\Delta \theta)} f_{\hat{\theta}}(t) d t .
\end{aligned}
$$

By the mean value theorem, we have

$$
\begin{aligned}
\left(\frac{\partial v\left(\theta, \omega^{\star}\right)}{\partial \theta} * 2 \Delta \theta+o(\Delta \theta)\right) \inf _{\hat{\theta} \in(\theta-\Delta \theta, \theta+\Delta \theta)} f_{\hat{\theta}}\left(v_{1}\right) & \leq \int_{0}^{v\left(\theta+\Delta \theta, \omega^{\star}\right)} f_{\theta+\Delta \theta}(t) d t+\int_{v\left(\theta-\Delta \theta, \omega^{\star}\right)}^{0} f_{\theta-\Delta \theta}(t) d t \\
& \leq\left(\frac{\partial v\left(\theta, \omega^{\star}\right)}{\partial \theta} * 2 \Delta \theta+o(\Delta \theta)\right) \sup _{\hat{\theta} \in(\theta-\Delta \theta, \theta+\Delta \theta)} f_{\hat{\theta}}\left(v_{2}\right),
\end{aligned}
$$


where $v_{1} \in\left(v\left(\theta-\Delta \theta, \omega^{\star}\right), v\left(\theta+\Delta \theta, \omega^{\star}\right)\right)$ and $v_{2} \in\left(v\left(\theta-\Delta \theta, \omega^{\star}\right), v\left(\theta+\Delta \theta, \omega^{\star}\right)\right)$, and $o(\Delta \theta)$ represents higher order terms of $\Delta \theta$.

Then we have

$$
\begin{aligned}
\Pi_{2} & =E\left[\psi(\theta) 1_{\{v(\theta) \geq 0\}} \mid v(\theta)=0\right] \frac{\partial v\left(\theta, \omega^{\star}\right)}{\partial \theta} \\
& =E\left[\psi(\theta) v^{\prime}(\theta) \mid v(\theta)=0\right]
\end{aligned}
$$

since $\lim _{\Delta \theta \rightarrow 0} \inf _{\hat{\theta} \in(\theta-\Delta \theta, \theta+\Delta \theta)} f_{\hat{\theta}}\left(v_{1}\right)=\lim _{\Delta \theta \rightarrow 0} \sup _{\hat{\theta} \in(\theta-\Delta \theta, \theta+\Delta \theta)} f_{\hat{\theta}}\left(v_{2}\right)=f_{\theta}(0)$.

Now consider $\Pi_{1}$. Note that for sufficiently small $\Delta \theta$,

$$
\begin{aligned}
& \left|\psi(\theta+\Delta \theta) 1_{\{v(\theta+\Delta \theta) \geq 0\}}-\psi(\theta-\Delta \theta) 1_{\{v(\theta-\Delta \theta) \geq 0\}}\right| \mathscr{B}^{c}\left(\omega^{\star}, \theta, \Delta \theta\right) \mid \\
\leq & |\psi(\theta+\Delta \theta)-\psi(\theta-\Delta \theta)| \mathscr{B}^{c}\left(\omega^{\star}, \theta, \Delta \theta\right) \mid \\
\leq & \lambda_{\psi(\theta)}|\Delta \theta| \quad \text { w.p.1. }
\end{aligned}
$$

The last inequality follows by Assumption 2. Then by the dominated convergence theorem,

$$
\lim _{\Delta \theta \rightarrow 0} \frac{E\left[\psi(\theta+\Delta \theta) 1_{\{v(\theta+\Delta \theta) \geq 0\}}-\psi(\theta-\Delta \theta) 1_{\{v(\theta-\Delta \theta) \geq 0\}} \mid \mathscr{B}^{c}\left(\omega^{\star}, \theta, \Delta \theta\right)\right]}{2 \Delta \theta}=E\left[\psi^{\prime}(\theta) 1_{\{v(\theta) \geq 0\}}\right] .
$$

Therefore, $\Pi_{1}=E\left[\psi^{\prime}(\theta) 1_{\{v(\theta)\} \geq 0}\right]$, and the theorem is proved.

\subsubsection{A Special Case}

Now consider a special case of the above theorem. Let $v(\theta)=U-\phi(\theta)$, where $U$ is a random variable independent of $\theta$ with p.d.f. $f_{U}$ and $\phi(\cdot)$ is a deterministic function. If $\phi(\cdot)$ is Lipschitz continuous and differentiable, then it is easy to see that $v(\theta)$ satisfies Assumption 1, so if Assumption 2 is also satisfied, then by Theorem 1, the estimator (6) becomes

$$
\frac{\partial}{\partial \theta} E\left[\psi(\theta) 1_{\{v(\theta) \geq 0\}}\right]=E\left[\psi^{\prime}(\theta) 1_{\{U \geq \phi(\theta)\}}\right]-f_{U}(\phi(\theta)) E\left[\psi(\theta) \phi^{\prime}(\theta) \mid U=\phi(\theta)\right] .
$$

\section{SENSITIVITY ANALYSIS FOR BARRIER OPTIONS}

Now we come back to sensitivity analysis for continuously-monitored barrier options. First we analyze the pricing problem for "standard" barrier options, based on which we derive a gradient estimator using the SPA estimator. To price the barrier option following the general diffusion model given by (2), we use the discretized approximation given by (3). We generate sample paths by (3) and compare the prices of the option at time $t_{1}, t_{2}, \cdots, t_{m}$ with the threshold value $H$ along a simulated path. If any of the prices at $t_{1}, t_{2}, \cdots, t_{m}$ is smaller than $H$ on a given sample path, then obviously the stock price has crossed the barrier, and the option payoff along that given sample path is zero. However, even if all the prices at the discretely sampled points are above $H$ on a given sample path, it is still possible that the stock price path crossed the barrier between two consecutive sampling points by going below and then coming back above. Thus, we need to incorporate this possibility in our analysis.

\subsection{Barrier Option Pricing}

Consider the stock price $S_{i-1}>H$ and $S_{i}>H$ at times $t_{i-1}$ and $t_{i}$, respectively. By (3), we know that the stock price follows a diffusion process with the drift term $\mu\left(S_{i-1}, t\right) d t$ and the Brownian motion term $d B_{t}$. Let $L_{i}=\inf _{t_{i-1}<t \leq t_{i}} S_{t}$. Then the option price can be computed as

$$
V=E\left[\prod_{i=1}^{m} 1_{\left\{L_{i}>H\right\}}\left(S_{m}-K\right)^{+}\right] .
$$


Given two values $S_{i-1}$ and $S_{i}$, then $S_{t}$ for $t \in\left(t_{i-1}, t_{i}\right]$ is a Brownian bridge and $L_{i}$ is the minimum of this Brownian bridge. By ?, we have

$$
P\left(L_{i}>H \mid S_{i-1}, S_{i}\right)=1_{\left\{S_{i}>H\right\}} \psi_{i}\left(H, S_{i-1}, S_{i}\right)
$$

where $\psi_{i}\left(H, S_{i-1}, S_{i}\right)=\left(1-\exp \left(-\frac{2\left(S_{i-1}-H\right)\left(S_{i}-H\right)}{\left(t_{i}-t_{i-1}\right) \sigma^{2}}\right)\right)$. Therefore, $1_{\left\{L_{i}>H\right\}}$ is equivalent to $1_{\left\{S_{i}>H\right\}} 1_{\left\{U_{i}<\psi_{i}\right\}}$, where $U_{i}$ is a random variable uniformly distributed in $[0,1]$ and $\psi_{i}$ is the abbreviation of $\psi_{i}\left(H, S_{i-1}, S_{i}\right)$. Hence we can rewrite the option price as

$$
V=E\left[\prod_{i=1}^{m} 1_{\left\{S_{i}>H\right\}} 1_{\left\{U_{i}<\psi_{i}\right\}}\left(S_{m}-K\right)^{+}\right]
$$

which also gives us an algorithm to approximate the option price.

Now we can use Theorem 1 to obtain the sensitivity of the option price with respect to a parameters of interest $\theta$ :

$$
\begin{aligned}
\frac{\partial V}{\partial \theta}= & E\left[\prod_{i=1}^{m} 1_{\left\{S_{i}>H\right\}} 1_{\left\{U_{i}<\psi_{i}\right\}} \frac{\partial\left(S_{m}-K\right)^{+}}{\partial \theta}\right]+\sum_{i=1}^{m} f_{\{i, H\}}(0) \\
& E\left[\prod_{j=1, j \neq i}^{m} 1_{\left\{S_{j}>H\right\}} 1_{\left\{U_{j}<\psi_{j}\right\}} 1_{\left\{U_{i}<\psi_{i}\right\}}\left(S_{m}-K\right)^{+} \mid S_{i}-H=0\right]+ \\
& \sum_{i=1}^{m} \frac{\partial \psi_{i}}{\partial \theta} E\left[\prod_{j=1, j \neq i}^{m} 1_{\left\{S_{j}>H\right\}} 1_{\left\{U_{j}<\psi_{j}\right\}} 1_{\left\{S_{i}>H\right\}}\left(S_{m}-K\right)^{+} \mid U_{i}=\psi_{i}\right] \\
= & E\left[\prod_{i=1}^{m} 1_{\left\{S_{i}>H\right\}} 1_{\left\{U_{i}<\psi_{i}\right\}} \frac{\partial\left(S_{m}-K\right)^{+}}{\partial \theta}\right]+ \\
& \sum_{i=1}^{m} \frac{\partial \psi_{i}}{\partial \theta} E\left[\prod_{j=1, j \neq i}^{m} 1_{\left\{S_{j}>H\right\}} 1_{\left\{U_{j}<\psi_{j}\right\}} 1_{\left\{S_{i}>H\right\}}\left(S_{m}-K\right)^{+} \mid U_{i}=\psi_{i}\right]
\end{aligned}
$$

where $f_{\{i, H\}}$ is the p.d.f. of the random variable $S_{i}-H$ for $i=1, \cdots, m$. The second equality holds because $\psi_{i}\left(H, S_{i-1}, H\right)=0$. We can condition on a small set where the value of payoff function changes and then take limit to get the result. The details of the derivation of the above gradient estimator are omitted.

\subsection{Barrier Option with Rebates}

Now we consider the pricing and sensitivity analysis for a type of barrier option with rebates. The buyers of this type of option will receive a rebate $\mathscr{R}$ if the option is knocked out, and the rebate is payable at the time of knock-out, so the value of the rebate is the product of the constant rebate value and a discount factor. In order to compute the expected value of the rebate, we need to know the distribution of the time when the stock price crosses the barrier. Define

$$
C(t):=\left\{\omega: \inf _{\tau} S_{\tau}(\omega) \leq H, \tau \in[t, t+d t]\right\}
$$

The p.d.f. of the first time when the stock price crosses the barrier between two end points is given by (Metwally and Atiya 2002)

$$
\begin{aligned}
g_{i}(t)= & P\left(C(t) \mid S_{i-1}, S_{i}\right) \\
= & \frac{S_{i-1}-H}{2 y\left(t_{i}-t_{i-1}\right) \sigma^{2}}\left(t-t_{i-1}\right)^{-3 / 2}\left(t_{i}-t\right)^{-1 / 2} \\
& \exp \left(-\left[\frac{\left(S_{i}-H-\mu\left(t_{i}-t\right)\right)^{2}}{2\left(t_{i}-t\right) \sigma^{2}}+\frac{\left(S_{i-1}-H+\mu\left(t-t_{i-1}\right)\right)^{2}}{2\left(t-t_{i-1}\right) \sigma^{2}}\right]\right)
\end{aligned}
$$


where

$$
y=\frac{1}{\sqrt{2 \pi\left(t_{i}-t_{i-1}\right)} \sigma} \exp \left(-\frac{S_{i-1}-S_{i}+\mu\left(t_{i}-t_{i-1}\right)}{2 \sigma^{2}\left(t_{i}-t_{i-1}\right)}\right) .
$$

Define $\eta:=\min \left\{i: S_{j}>H, \forall j=1, \cdots, i-1 ; S_{i} \leq H\right\}$, which is the index of the time when the stock price crosses the barrier the first time. If the stock price never crosses the barrier, we let $\eta=0$. The payoff of the down-and-out barrier option along a simulated path can be computed as

$$
\begin{aligned}
\hat{V}= & \sum_{i=1}^{\Gamma} \mathscr{R} \prod_{j=1}^{i-1} 1_{\left\{S_{j}>H\right\}} 1_{\left\{U_{j}<\psi_{j}\right\}} 1_{\left\{U_{i}>\psi_{i}\right\}} 1_{\left\{S_{i}>H\right\}} E_{p_{i}}\left[\exp \left(-r \tau_{i}\right)\right]+1_{\{\eta \neq 0\}} \mathscr{R} \prod_{j=1}^{\Gamma} 1_{\left\{S_{j}>H\right\}} 1_{\left\{U_{j}<\psi_{j}\right\}} 1_{\left\{S_{\Gamma+1} \leq H\right\}} \\
& E\left[\exp \left(-r \tau_{\Gamma+1}\right)\right]+1_{\{\eta=0\}} \exp (-r T)\left(S_{m}-K\right)^{+} \prod_{j=1}^{m} 1_{\left\{S_{j}>H\right\}} 1_{\left\{U_{j}<\psi_{j}\right\}},
\end{aligned}
$$

where $\Gamma=\eta-1$, if $\eta \neq 0$; $\Gamma=m$, if $\eta=0$; Let $\Lambda_{i}$ be the event that the stock price crosses the barrier, given two end points $S_{i-1}, S_{i} . \tau_{i}=\left\{\inf t: S_{t}=H \mid S_{i-1}, S_{i}, \Lambda_{i}\right\} ; p_{i}=P\left(C(s) \mid S_{i-1}, S_{i}, \Lambda_{i}\right) ;\left\{U_{j}, j=1, \cdots, \Gamma\right\}$ are uniformly distributed random variables in $[0,1]$, which are independent of each other and $\left\{S_{i}, i=1, \cdots, m\right\}$. Note that

$$
E_{p_{i}}\left[\exp \left(-r \tau_{i}\right)\right]=\int_{t_{i-1}}^{t_{i}} \exp (-r s) \frac{P\left(C(s) \mid S_{i-1}, S_{i}\right)}{P\left(\Lambda_{i}\right)} d s
$$

We can use the following algorithm to simulate the price of the barrier option with rebates.

\section{Algorithm 1.}

1 Initialization: let $n=1$.

2 If $n>N$, go to step 3. Otherwise, according to (3), simulate stock price $S_{i}, i=1, \cdots, m$ by generating standard normal distributed random variables $z_{i}, i=1, \cdots, m$. Let $i=1$, and perform the following iteration.

[2a] If $S_{i}>H$, generate $U_{i}$, which is uniformly distributed in $[0,1]$, and compute $\psi_{i}$, then go to step $2 \mathrm{~b}$; if $S_{i} \leq H$, let $\hat{V}_{n}=\int_{t_{i-1}}^{t_{i}} \exp (-r s) g_{i}(s) d s$. let $n=n+1$ and go to step 2 .

[2b] If $U_{i}<\psi_{i}$ and $i=m$, let $\hat{V}_{n}=\exp (-r T)\left(S_{m}-K\right)^{+}$; If $U_{i}<\psi_{i}$ and $i<m$, let $i=i+1$, go to step 2a. If $U_{i} \geq \psi_{i}$, let $\hat{V}_{n}=\int_{t_{i-1}}^{t_{i}} \exp (-r s) g_{i}(s) d s$. let $n=n+1$ and go to step 2 .

$3 \quad \bar{V}=\frac{1}{N} \sum_{i=1}^{N} \hat{V}_{i}$.

By the above algorithm, we can easily obtain an approximation of the option price using Monte Carlo simulation. Now we will use SPA technique to derive a gradient estimator for the barrier option with respect to parameters of interest. Note that the expectation of the payoff given by (8) is different from the function (4) considered in Theorem 1. For payoff (8), when we condition on a small set which contains the points where the value of the indicator functions change, the random variable $\Gamma$ changes, and hence changes the form of the payoff function. However, in Theorem 1, if the value of the indicator function becomes zero, the value of the payoff functions changes to zero.

It is not difficult to see that the payoff function (8) is continuous in $H$ at points $\left\{S_{i}=H\right\}$. We only need to use conditional expectation to smooth out the indicator function $1_{\left\{U_{j}<\psi_{j}\right\}}$. Following the same spirit as the method used to prove Theorem 1 , we have

$$
\frac{\partial E[\hat{V}]}{\partial H}=\Lambda_{1}-\Lambda_{2}+\lim _{\Delta H \rightarrow 0} o(\Delta H)
$$


where

$$
\begin{aligned}
\Lambda_{1}= & \mathscr{R} E\left[\sum_{i=1}^{\Gamma} \prod_{j=1}^{i-1} 1_{\left\{S_{j}>H\right\}} 1_{\left\{U_{j}<\psi_{j}\right\}} 1_{\left\{S_{i}>H\right\}} 1_{\left\{U_{i} \geq \psi_{i}\right\}} \frac{\partial}{\partial H} E_{p_{i}}\left[\exp \left(-r \tau_{i}\right)\right]\right]+ \\
\mathscr{R} & {\left[1_{\{\eta \neq 0\}} \prod_{j=1}^{\Gamma} 1_{\left\{S_{j}>H\right\}} 1_{\left\{U_{j}<\psi_{j}\right\}} 1_{\left\{S_{\Gamma+1} \leq H\right\}} \frac{\partial}{\partial H} E\left[\exp \left(-r \tau_{\Gamma+1}\right)\right]\right] } \\
\Lambda_{2}= & E\left[\sum _ { i = 1 } ^ { \Gamma } \frac { \partial \psi _ { i } } { \partial H } \left\{\mathscr{R} \prod_{j=1}^{i-1} 1_{\left\{S_{j}>H\right\}} 1_{\left\{U_{j}<\psi_{j}\right\}} 1_{\left\{S_{i}>H\right\}} E_{p_{i}}\left[\exp \left(-r \tau_{i}\right)\right]-\right.\right. \\
& \sum_{l=i+1}^{\Gamma} \prod_{j=1}^{l-1} 1_{\left\{S_{j}>H\right\}} 1_{\left\{U_{j}<\psi_{j}, j \neq i\right\}} 1_{\left\{U_{l} \geq \psi_{l}\right\}} 1_{\left\{S_{l}>H\right\}} E_{p_{l}}\left[\exp \left(-r \tau_{l}\right)\right]- \\
& 1_{\{\eta \neq 0\}} \mathscr{R} \prod_{j=1}^{\Gamma} 1_{\left\{S_{j}>H\right\}} 1_{\left\{U_{j}<\psi_{j}, j \neq i\right\}} 1_{\left\{S_{\Gamma+1} \leq H\right\}} E\left[\exp \left(-r \tau_{\Gamma+1}\right)\right]- \\
& \left.\left.1_{\{\eta=0\}} \exp (-r T)\left(S_{m}-K\right)^{+} \prod_{j=1}^{m} 1_{\left\{S_{j}>H\right\}} 1_{\left\{U_{j}<\psi_{j}, j \neq i\right\}} \mid U_{i}=\psi_{i}\right\}\right],
\end{aligned}
$$

and $o(\Delta H)$ are the higher order term of $\Delta H$. Since there are multiple indicator functions in (8), when we condition on small sets involving more than one discontinuous points, we have such higher order terms.

\subsection{Gradient Estimator}

When Algorithm 1 is used to price the barrier option, we need to estimate the integral $\int_{t_{i-1}}^{t_{i}} \exp (-r s) g_{i}(s) d s$, given two end points $S_{i-1}$ and $S_{i}$. It is not easy to sample from p.d.f. $g_{i}(s)$ directly. However through some algebraic operation, (7) becomes

$$
\begin{aligned}
g_{i}(t)= & \frac{S_{i-1}-H}{\sqrt{2 \pi\left(t-t_{i}\right)^{3} \sigma^{2}}} \sqrt{\frac{t_{i}-t_{i-1}}{t_{i}-t}} \\
& \exp \left(-\frac{\left[\left(S_{i-1}-H-\left(S_{i}-H\right)\right)\left(t-t_{i-1}\right)-\left(S_{i-1}-H\right)\left(t_{i}-t_{i-1}\right)\right]^{2}}{2\left(t_{i}-t_{i-1}\right)\left(t-t_{i-1}\right)\left(t_{i}-t\right) \sigma^{2}}\right) .
\end{aligned}
$$

Note that the inverse Gaussian process random variable has the following p.d.f.:

$$
f_{i}\left(y, \eta_{i}, \lambda_{i}\right)=\sqrt{\frac{\lambda_{i}}{2 \pi y^{3}}} \exp \left(\frac{-\lambda_{i}\left(y-\eta_{i}\right)^{2}}{2 \eta_{i}^{2} y}\right),
$$

which has a similar form with (10). It is not difficult to show that if a random variable $Y$ has density $f_{i}\left(y, \eta_{i}, \lambda_{i}\right)$ with $\eta_{i}=-\frac{S_{i-1}-H}{S_{i}-H}$ and $\lambda_{i}=\frac{\left(S_{i-1}-H\right)^{2}}{\left(t_{i}-t_{i-1}\right) \sigma^{2}}$, then $t_{i-1}+\frac{\left(t_{i}-t_{i-1}\right) Y}{1+Y}$ has density (7). Therefore $\int_{t_{i-1}}^{t_{i}} \exp (-r s) g_{i}(s) d s$ can be easily approximated using Monte Carlo simulation by generating samples of the random variable $Y$ (Glasserman 2004).

To obtain the gradient estimator (9), we need to estimate the gradient of $\int_{t_{i-1}}^{t_{i}} \exp (-r s) g_{i}(s) d s$ with respect to $H$. Since $H$ only exists in the probability density function $g_{i}(s)$, we can obtain a likelihood ratio (LR) gradient estimator easily, which is given as follows:

$$
\begin{aligned}
\frac{\partial}{\partial H} \int_{t_{i-1}}^{t_{i}} \exp (-r s) g_{i}(s) d s & =\frac{\partial}{\partial H} \int_{0}^{\infty} \exp \left(-r t_{i-1}+\frac{\left(t_{i}-t_{i-1}\right) y}{1+y}\right) f_{i}\left(y, \eta_{i}, \lambda_{i}\right) d y \\
& =\int_{0}^{\infty} \exp \left(-r\left(t_{i-1}+\frac{\left(t_{i}-t_{i-1}\right) y}{1+y}\right)\right) \frac{\partial \ln f_{i}\left(y, \eta_{i}, \lambda_{i}\right)}{\partial H} f_{i}\left(y, \eta_{i}, \lambda_{i}\right) d y
\end{aligned}
$$


Wang, Fu and Marcus

Table 1: Sensitivity Estimation Result for Barrier Options without Rebates (standard errors in parentheses)

\begin{tabular}{ccccc}
\hline \hline \multicolumn{5}{c}{$r=0.125, \sigma=0.5, K=100, H=98$} \\
$\partial V / \partial H$ & $m=5$ & $m=10$ & $m=15$ & $m=20$ \\
\hline Exact & -1.293 & -1.293 & -1.293 & -1.293 \\
FD & $-1.11(0.45)$ & $-0.92(0.40)$ & $-0.92(0.42)$ & $-0.87(0.53)$ \\
SPA & $-1.292(0.04)$ & $-1.24(0.04)$ & $-1.377(0.05)$ & $-1.312(0.05)$ \\
\hline \hline
\end{tabular}

Table 2: Sensitivity Estimation Result for Barrier Options with Rebates (standard errors in parentheses)

\begin{tabular}{cccccc}
\hline \hline \multicolumn{7}{c}{$r=0.02, \sigma=0.5, K=100, H=95$} \\
$\partial V / \partial H$ & $m=10$ & $m=20$ & $m=40$ & $m=80$ & $m=160$ \\
\hline FD & $-2.63(0.8)$ & $-2.09(0.5)$ & $-1.77(0.6)$ & $-1.57(0.5)$ & $-1.86(0.6)$ \\
SPA & $-2.14(0.03)$ & $-2.11(0.06)$ & $-2.26(0.08)$ & $-2.22(0.09)$ & $-2.11(0.1)$ \\
\hline \hline
\end{tabular}

\section{NUMERICAL RESULTS}

In this section, we give numerical results for two examples to demonstrate the efficiency of the estimators. For simplicity, we consider a Black-Scholes model with parameters $S_{0}=K=100, r=0.125, \sigma=0.5, T=1$, and $H=98$. For the Black-Scholes model, we can obtain the exact gradient values for all parameters of interest. We compare the performance of our estimator with the exact values and also with finite difference estimates. When we derive the finite difference estimator, we make a fine discretization of the stochastic process that governs the stock price: $\left\{S_{t_{i}}, i=1, \cdots, m_{F}\right\}$, where $m_{F}$ is the number of the discretized points. The discretized time step is $T / m_{F}=2 * 10^{-4}$. We use $\min _{i=1, \cdots, m_{F}} S_{t_{i}}$ to approximate $\inf _{t \leq T} S_{t}$ and generate $N=10000$ sample paths to get one estimate. From the simulation results, we can see that the SPA gradient estimator generally works well, whereas the finite difference gradient estimator has bias with comparatively larger variance. Moreover from the simulation result, we can see the performance of our gradient estimator doesn't change a lot with the changing of $m$, which is the number of discretization points. Since $H$ is in the indicator function, there will be an abrupt change in the performance function when the change of $H$ reaches a certain level. Therefore the finite difference estimator tends to have larger variance.

In our second example, we consider sensitivity analysis for barrier options with rebates. For simplicity, we also consider Black-Scholes model with parameters $S_{0}=K=100, r=0.02, \sigma=0.5, T=1, H=95$, and $\mathscr{R}=5$. Even for this Black-Scholes model, it is not clear whether there is an analytical solution. Therefore we mainly compare our result with the finite difference gradient estimator. We obtain the finite difference gradient estimator by discretizing the stochastic process with a very small time step $h=2 * 10^{-4}$. The first discretized time when the stock price crosses the barrier will be the time when the rebate is paid. If there is no barrier crossing before $T$, we get payoff $\exp (-r T)\left(S_{m}-K\right)^{+}$. We generate $N=10000$ sample paths to get one estimate. As in the previous example, the SPA estimator is clearly superior to the FD estimator in terms of precision, and the difference is even wider, as the standard error of the FD estimate has increased.

\section{CONCLUSIONS}

Motivated by the sensitivity analysis problem for barrier options, this paper explores the connection between SPA and the gradient estimator derived in (Hong 2008), which involves construction of auxiliary variables. Specifically, a general class of sample performance functions with an indicator function discontinuity is considered. By conditioning on a small set (interval) that contains the discontinuity point, we can smooth out the discontinuity of the sample performance function and thus obtain an unbiased pathwise gradient estimator. A drawback often cited in applying SPA to a specific problem setting is the difficulty of choosing what set to condition on. Here, we demonstrate that for the setting of a sample performance function with a given point of discontinuity, we only need to condition on a small set around the discontinuity point and then take the appropriate limit. Furthermore, this approach can be generalized beyond the form of the sample performance function consider in Theorem 1. Here, we consider continuously-monitored barrier options and in conjunction with a Brownian bridge construction, apply the approach to derive gradient estimators for the basic case and then generalize the estimator to barrier options with rebates. Numerical results indicate that our estimators work well. 
Wang, Fu and Marcus

\section{ACKNOWLEDGMENTS}

This work was supported in part by the National Science Foundation (NSF) under Grants DMI-0540312 and DMI-0323220, and by the Air Force Office of Scientific Research (AFOSR) under Grant FA9550-07-1-0366.

\section{REFERENCES}

Boyle, P. P. 1977. Options: A Monte Carlo approach. Journal of Financial Economics 4:323-338.

Broadie, M., and P. Glasserman. 1996. Estimating security price derivatives using simulation. Management Science 42 (2): 269-285.

Broadie, M., P. Glasserman, and S. Kou. 1997. A continuity correction for discrete barrier option prices. Mathematical Finance 7:325-349.

Chen, N., and P. Glasserman. 2007. Malliavin Greeks without Malliavin calculus. Stochastic Processes and their Applications 117:1689-1723.

Fournié, E., J.-M. Lasry, J. Lebuchoux, and P.-L. Lions. 2001. Applications of Malliavin calculus to Monte Carlo methods in finance II. Finance and Stochastics 5:201-236.

Fu, M. C. 2006. Gradient estimation. In Handbooks in Operations Research and Management Science: Simulation, ed. S. G. Henderson and B. L. Nelson, Chapter 19, 575-616. Elsevier.

Fu, M. C., and J. Q. Hu. 1995. Sensitivity analysis for Monte Carlo simulation of option pricing. Probability in the Engineering and Informational Sciences 9 (3): 417-446.

Fu, M. C., and J. Q. Hu. 1997. Conditional Monte Carlo: Gradient estimation and optimization applications. Kluwer Academic Publishers.

Glasserman, P. 1991. Gradient estimation via perturbation analysis. Kluwer Academic Publishers, Boston, Massachusetts.

Glasserman, P. 2004. Monte Carlo methods in financial engineering. Springer, New York.

Glasserman, P., and J. Staum. 2001. Conditioning on one-step survival for barrier option simulations. Operations Research 49 (6): 923-937.

Gong, W. B., and Y. C. Ho. 1987. Smoothed perturbation analysis of discrete-event dynamic systems. IEEE Transactions on Automatic Control AC-32:858-867.

Ho, Y. C., and X. R. Cao. 1991. Perturbation analysis and discrete event dynamic systems. Kluwer Academic.

Hong, L. J. 2008. Estimating quantile sensitivities. Operations Research 57:118-130.

Karatzas, I., and S. Shreve. 1991. Brownian motion and stochastic calculus. New York: Springer-Verlag.

Liu, G., and J. Hong. 2009. Pathwise estimation of the Greeks of financial options. submitted.

Metwally, S. A., and A. F. Atiya. 2002. Using Brownian bridge for fast simulation of jump-diffusion processes and barrier options. The Journal of Derivatives 10 (1): 43-54.

Reiman, M., and A. Weiss. 1989. Sensitivity analysis for simulations via likelihood ratios. Operations Research 37:830-844. Rubinstein, R., and B. Melamed. Modern simulation and modeling. Wiley Series in Probability and Statistics.

\section{AUTHOR BIOGRAPHIES}

YONGQIANG WANG is a Ph.D. student in the Department of Electrical and Computer Engineering and the Institute for Systems Research, University of Maryland. He received the B.S. degree (with highest honor) in electrical engineering and M.E. degree from Zhejiang University in 2003 and 2006, respectively. He has been selected as a Future Faculty Fellow by the A. J. Clark School of Engineering at the University of Maryland. His research interests lie in the areas of simulation based optimization, Markov decision process, and stochastic control, with applications towards supply chain management and financial engineering. His email address is $\left\langle y q w a n g @ u m d\right.$.edu>, and personal website www.ece.umd.edu/ ${ }^{\text {yqwang. }}$

MICHAEL C. FU is Ralph J. Tyser Professor of Management Science in the Robert H. Smith School of Business, with a joint appointment in the Institute for Systems Research and an affiliate appointment in the Department of Electrical and Computer Engineering, all at the University of Maryland. He received degrees in mathematics and EE/CS from MIT, and an M.S. and Ph.D. in applied mathematics from Harvard University. His research interests include simulation and applied probability modeling, particularly with applications towards manufacturing systems, supply chain management, and financial engineering. He has served as Stochastic Models and Simulation Department Editor of Management Science and as Simulation Area Editor of Operations Research. He is co-author (with J.Q. Hu) of the book, Conditional Monte Carlo: Gradient Estimation and Optimization Applications, which received the INFORMS Simulation Society's Outstanding Publication Award in 1998. 
He is a Fellow of INFORMS and IEEE. His email address is $<$ mfueumd. edu $>$.

STEVEN I. MARCUS is a Professor in the Department of Electrical and Computer Engineering and the Institute for Systems Research, University of Maryland. He received the B.A. degree in electrical engineering and mathematics from Rice University in 1971, and the S.M. and Ph.D. degrees in electrical engineering from MIT in 1972 and 1975, respectively. From 1975 to 1991, he was with the Department of Electrical and Computer Engineering, University of Texas, Austin, where he was the L.B. (Preach) Meaders Professor in Engineering. At the University of Maryland, he has served as Director of the Institute for Systems Research from 1991-1996, and as Chair of the Electrical and Computer Engineering Department from 2000-2005. Currently, his research is focused on stochastic control and estimation, with applications in manufacturing and telecommunication networks. He is the Corresponding Editor of the SIAM Journal on Control and Optimization and a Fellow of IEEE and SIAM. His email address is <marcuseumd. edu>. 